\title{
Avaliação reológica e sensorial de espessantes domésticos em diferentes líquidos como alternativa na disfagia
}

\author{
Sensory and rheological characteristics of homemade thickeners in different liquids \\ as an alternative in cases of dysphagia
}

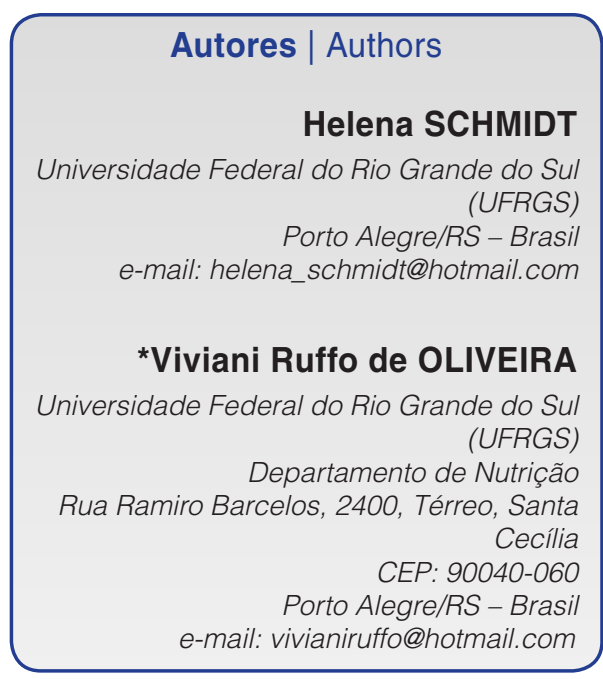

*Autor Correspondente / Corresponding Author

Recebido: Jul. 22, 2014

Aprovado: Abr. 01, 2015

\section{Resumo}

O objetivo desse estudo foi analisar as propriedades reológicas e a aceitabilidade de espessantes domésticos em diferentes líquidos, além de comparar os custos e o valor energético desses espessantes. Avaliaram-se no viscosímetro de Brookfield amostras à base de farinha de fécula de batata, de amido de milho, de polvilho doce e de farinha de arroz, além de dois espessantes comerciais, diluídos em líquidos. Foi realizada, com a participação de 30 avaliadores, a investigação de aceitabilidade dos espessantes em água, leite e suco para os atributos: aparência, consistência, odor, sabor e aceitação global. A viscosidade em água dos espessantes comerciais, do amido de milho e da farinha de arroz não teve diferença estatística significativa entre si. Um dos espessantes comerciais atingiu a consistência "rala". Os resultados de leites e sucos foram semelhantes quando classificados por consistência. $\mathrm{Na}$ aceitabilidade dos atributos odor e sabor para água, o espessante comercial, a fécula de batata, o polvilho doce e a farinha de arroz não apresentaram diferença significativa entre si. No leite, para os atributos odor e sabor as amostras não tiveram diferença significativa entre si e para sucos, o espessante comercial, a fécula de batata e o polvilho doce não apresentaram diferença significativa. A viscosidade de um dos espessantes comerciais não estava adequada para o consumo por pacientes com disfagia, obtendo consistência "rala". O espessante comercial E.C1F e o amido de milho (AM) apresentaram consistência "mel", sendo apropriado para o consumo em líquidos. As amostras com leites foram as que apresentaram maiores viscosidades, a maioria obteve consistência "pudim". A aceitabilidade da E.C1F e da fécula de batata (FB) em leite foram as que mais se destacaram promissoras.

Palavras-chave: Disfagia; Espessantes; Viscosidade; Análise sensorial.

\section{Summary}

The objective of this study was to evaluate the sensory and rheological characteristics of homemade thickeners in different liquids as an alternative for patients with dysphagia, besides comparing their prices and energy values. Samples based on potato starch flour, corn starch, cassava starch, rice flour and two commercial thickeners diluted in different liquids were evaluated using a Brookfield viscometer. The sensory acceptability of the thickeners in water, milk and juice was evaluated by 30 judges for the attributes of appearance, consistency, odour, flavour and global acceptance. The viscosities of the commercial thickeners in water and of the corn starch and rice flour in water showed no statistically significant differences. With respect to consistency, one of the commercial thickeners was classified as "thin". The results for the consistency of the samples in milk and juice were all similar. In the sensory analysis for the attributes of odour and flavour of the samples in water, the commercial thickener, potato starch, cassava starch and rice flour showed no statistical differences. In milk, the samples showed no significant differences for the attributes of odour and flavour, and in juice, the commercial thickener, potato starch and cassava starch showed no significant differences. The viscosity of one of the commercial thickeners was not suitable for consumption by dysphagia patients due to its "thin" consistency. The commercial thickener E.C1F and the corn starch showed a consistency of "honey", being suitable for fluid intake. The milk samples showed the highest viscosities, the consistency of the majority being classified as "pudding". The acceptability of the E.C1F and potato starch samples in milk stood out as being the most promising.

Key words: Dysphagia; Thickeners; Viscosity; Sensory analysis. 


\section{Introdução}

A disfagia pode ser resultado de alterações neurológicas e estruturais causadas por problemas na cavidade oral, na faringe, no esôfago e/ou em junções gastroesofageanas (SCHELP et al., 2004). A dificuldade de deglutição traz consigo o risco de causar engasgos, sufocamento, aspiração, problemas pulmonares, deficiências nutricionais, perda de peso, e desidratação (SCHELP et al., 2004).

Segundo SBGG (2011), a disfagia exige modificação da dieta, suplementação nutricional e espessamento de líquidos, dentre outras manobras. É justamente o espessamento de preparações que implica em um importante papel para melhorar a adequação do estado nutricional das pessoas acometidas (SILVA e IKEDA, 2009).

A viscosidade do alimento é uma das variáveis mais importantes na deglutição. Os líquidos ralos dificultam a deglutição de pacientes que apresentam controle laríngeo reduzido (SOUZA et al., 2003). Modificações na viscosidade dos alimentos e fluidos podem ser conseguidas com o auxílio de espessantes industrializados, porém, esses alimentos são considerados de alto custo e, além disso, não são facilmente encontrados em estabelecimentos comerciais, limitando a aquisição e ajuste da consistência correta. O espessamento a partir da utilização dos próprios alimentos em preparações diversas para ajuste da consistência alimentar é ainda pouco conhecido por muitos dos pacientes, cuidadores e profissionais da saúde (SILVA e IKEDA, 2009).

Entretanto, o uso de diferentes espessantes pode conferir viscosidade com diferentes graus de aceitação pelos consumidores, devido à textura percebida ao degustarem esses produtos. Para identificar qual espessante se ajusta melhor ao produto é necessária uma avaliação sensorial (FONTAN, 2008).

Os principais tipos de espessantes podem ser divididos em duas classes: as gomas e os amidos. A classe dos amidos pode ser dividida em: amidos e amidos modificados. Dentre os amidos modificados comercializados, encontram-se suplementos que são usualmente utilizados em hospitais, como o Thick \& Easy ${ }^{\otimes}$ (Hormel/ Health Labs/ Fresenius) e o
Resource Ticken Up ${ }^{\circledR}$ (Novartis $^{\circledast}$ ) e Nutilis ${ }^{\circledast}\left(\right.$ Support $\left.^{\circledR}\right)$. Esses suplementos são bem-aceitos, pois não alteram o sabor e a cor dos alimentos, e oferecem uma média de $18 \mathrm{kcal}$ a cada $5 \mathrm{~g}$ (SOUZA et al., 2003).

Promover a nutrição adequada e a viscosidade ideal na disfagia é uma dificuldade que necessita ser superada, uma vez que a alimentação desses indivíduos pode ser facilitada se os alimentos tiverem a textura modificada para "pudim" e principalmente se os líquidos forem espessados. No entanto, essas mudanças frequentemente resultam em alimentos que são menos atraentes sensorialmente (GERMAIN et al., 2006), constituindo um grande desafio.

Sendo assim, em função da necessidade do cuidado nutricional na disfagia esse estudo tem como objetivo analisar as propriedades reológicas, a aceitabilidade de espessantes em diferentes líquidos, além de comparar os custos e valor energético dos espessantes domésticos e comerciais avaliados.

\section{Material e métodos}

\subsection{Preparo das amostras}

Os alimentos e os espessantes utilizados foram adquiridos em comércio local da cidade de Porto Alegre, RS. Para a seleção das amostras utilizaram-se farinhas à base de amido de maior uso doméstico e/ou acessibilidade comercial. Os testes preliminares para os ajustes de formulações e o preparo das amostras foram realizados no Laboratório de Técnica Dietética do curso de Nutrição da Faculdade de Medicina da Universidade Federal do Rio Grande do Sul (UFRGS). Para a preparação dos espessantes, todos os ingredientes foram medidos usando materiais específicos para medidas caseiras tais como: balança semi-analítica, marca Shimadzu ${ }^{\circledR}$ Classe II, BL $3200 \mathrm{H}$; colheres padronizadas e beckers, e quando foram utilizados espessantes comercializados, seguiu-se as orientações de quantidade e forma de preparo do fabricante. Foram utilizados os seguintes espessantes oriundos de farinhas de alimentos: F.B - Fécula de batata; A.M - Amido de milho; P.D- Polvilho doce; F.A- Farinha de arroz, e dois espessantes comerciais: E.C1- Espessante comercial 1 e E.C2- Espessante comercial 2; suas viscosidades foram avaliadas conforme Tabela 1.

Tabela 1. Consistências dos produtos, viscosidade, descrição e exemplos.

\begin{tabular}{ccll} 
Consistência & Viscosidade (cP) & \multicolumn{1}{c}{ Descrição da Consistência } & \multicolumn{1}{c}{ Exemplos } \\
Rala & $1-50$ & Líquidos ralos & Água, gelatina, café, chás, sucos, refrigerante \\
Néctar & $51-350$ & O líquido escorre da colher formando um fio & Suco de manga ou pêssego ou iogurte de beber \\
Mel & $351-1750$ & O líquido escorre da colher formando um V & Mel \\
Pudim & $>1750$ & $\begin{array}{l}\text { O líquido escorre da colher, caindo em } \\
\text { bloco }\end{array}$ & Creme de abacate e iogurtes cremosos
\end{tabular}

Fonte: Adaptada de Crary et al. (2005), NDD (2002). 
Os líquidos utilizados foram: água filtrada, suco de laranja industrializado e leite desnatado UHT. Para a preparação das amostras foram homogeneizados $200 \mathrm{~mL}$ de cada amostra dos líquidos + farinha em um recipiente de inox que foi submetido ao aquecimento no fogão. A amostra foi aquecida a $180^{\circ} \mathrm{C}$ sob agitação até atingir a gelatinização do amido, formando um gel ou pasta (MOURA e ASCHERI, 2013).

Os espessantes comerciais foram diluídos conforme especificado pelo fabricante e todas as amostras foram avaliadas em triplicata (Tabela 2).

Calculou-se o valor energético das amostras por meio da Tabela Brasileira de Composição de Alimentos (UNICAMP, 2006) e quando não constava na tabela, foi utilizado o rótulo do alimento. O preço dos produtos adquiridos foi o da nota fiscal na hora da aquisição.

\subsection{Viscosidade}

As medidas reológicas foram realizadas no Laboratório da Faculdade de Farmácia da Universidade Federal do Rio Grande do Sul (UFRGS) e analisadas em triplicata, sob $25+1^{\circ} \mathrm{C}$, empregando um viscosímetro rotatório, marca RVDVII+, Brookfield, US, com spindles $21 \mathrm{e}$ 29, conectado a um sistema computadorizado de aquisição de dados (Rheocal V.3.1-1) para registrar a tensão de cisalhamento $(\mathrm{Pa})$, a taxa de deformação (s-1) e a viscosidade aparente (Pas). A classificação para consistências em rala, néctar, mel e pudim estão na Tabela 1.

\subsection{Análise sensorial}

Após a análise reológica, foram selecionados para análise sensorial os cinco espessantes que se apresentaram mais promissores, ou seja, com viscosidade segura de acordo com a disfagia. Foi realizado um teste afetivo no Laboratório de Técnica Dietética do curso de
Nutrição da Faculdade de Medicina da Universidade Federal do Rio Grande do Sul (UFRGS) com 30 avaliadores compostos por alunos, docentes e funcionários da própria instituição, com idade entre 18-60 anos e de ambos os sexos, não-treinados, não-portadores de disfagia, com disponibilidade de tempo e motivação em participar; foram observados os seguintes atributos: aparência, consistência, odor, sabor e aceitação global.

As amostras foram avaliadas em copos brancos descartáveis, identificados com códigos de três dígitos aleatórios, seguindo o delineamento de blocos incompletos. Foi fornecido ainda, um copo de água para limpeza das papilas gustativas e uma ficha para avaliação, a qual continha uma escala hedônica de nove pontos: 1 - "desgostei muitíssimo", 2 - "desgostei muito", 3 - "desgostei moderadamente", 4 - "desgostei ligeiramente", 5 - "indiferente", 6 - "gostei ligeiramente", 7 - "gostei moderadamente", 8 - "gostei muito" e 9 - "gostei muitíssimo".

\subsection{Aspectos éticos}

Os participantes assinaram um Termo de Consentimento Livre e Esclarecido avaliado e aprovado pelo Comitê de Pesquisa FAMED/UFRGS e Ética da Universidade Federal do Rio Grande do Sul (UFRGS) conforme a Resolução 466/12 do CNS/MS (BRASIL, 2013) e registrado sob protocolo $n^{\circ} 15.766$.

\subsection{Análise estatística}

Os resultados obtidos foram avaliados por meio de análise de variância e para comparação das médias foi realizado o Teste de Tukey. Os resultados das análises reológicas e sensoriais foram calculados com o nível de significância de 5\% de probabilidade de erro no software estatístico ESTAT versão 2.0 (ESTAT, 1992).

Tabela 2. Espessantes comerciais e domésticos utilizados na análise reológica, quantidade (g) e medida caseira diluídas em água, leite e suco.

\begin{tabular}{cccccc} 
Amostras & \multicolumn{2}{c}{ Quantidade $(\mathbf{g})$} & Medida caseira & $\begin{array}{c}\text { Quantidade }(\mathbf{g}) \\
\text { Suco }\end{array}$ & Medida caseira \\
\hline E.C1F & 6 & 6 & 4 medidas & 6 & 4 medidas \\
E.C1 & 10 & 10 & 7 medidas & 8 & 5 medidas \\
E.C2F & 6 & 6 & 2 medidas & 6 & 2 medidas \\
E.C2 & 10 & 10 & $31 / 2$ medidas & 8 & 3 medidas \\
FB & 10 & 10 & 2 colheres de chá niveladas & 8 & 2 colheres de chá niveladas \\
PD & 10 & 10 & $31 / 2$ colheres de chá niveladas & 8 & 3 colheres de chá niveladas \\
AM & 10 & 10 & 3 colheres de chá niveladas & 8 & 3 colheres de chá niveladas \\
FA1 & 10 & 10 & 2 colheres de chá niveladas & 8 & 1 1 $1 / 2$ colher de chá niveladas \\
FA2 & 20 & 20 & 5 colheres de chá niveladas & 16 & $31 / 2$ colheres de chá niveladas \\
\hline
\end{tabular}

E.C1F: Espessante comercial 1 diluído conforme o fabricante. E.C1: Espessante comercial $1 \mathrm{com} 10 \mathrm{~g}$ para água e leite e $8 \mathrm{~g}$ para suco. E.C2F: Espessante comercial 2 diluído conforme o fabricante. E. C2: Espessante comercial $2 \mathrm{com} 10 \mathrm{~g}$ para água e leite e $8 \mathrm{~g}$ para suco. FB Fécula de Batata com $10 \mathrm{~g}$ para água e leite e $8 \mathrm{~g}$ para suco. PD: Polvilho doce com $10 \mathrm{~g}$ para água e leite e $8 \mathrm{~g}$ para suco. AM: Amido de milho com $10 \mathrm{~g}$ para água e leite e $8 \mathrm{~g}$ para suco. FA1: Farinha de arroz com $10 \mathrm{~g}$ para água e leite e $8 \mathrm{~g}$ para suco. FA2: Farinha de arroz com $20 \mathrm{~g}$ para água e leite e $16 \mathrm{~g}$ para suco. 


\section{Resultados e discussão}

As medidas de viscosidades realizadas com os diferentes espessantes para água, leite e suco podem ser observadas na Tabela 3.

De acordo com a Tabela 3, na água, o Espessante Comercial E.C1F com 6g, o E.C1 com $10 \mathrm{~g}$ e o amido de milho ( $A M)$ apresentaram consistência "mel" (351-1750cP). O Espessante Comercial E.C2 quando mais concentrado (10g), a fécula de batata (FB) e o polvilho doce (PD) com $10 \mathrm{~g}$ apresentaram consistência "pudim" (>1750 cP). A farinha de arroz (FA) com 10g atingiu a consistência "néctar" (51-350 cP), porém quando dobrada a sua quantidade (FA2), atingiu a consistência de "pudim".

Para as amostras com leites apenas o Espessante Comercial E.C2F diluído conforme as orientações do fabricante e a farinha de arroz com $10 \mathrm{~g}$ ficaram na consistência "néctar", enquanto que nas demais amostras, a consistência alcançada foi a de "pudim". Dentre as amostras analisadas o leite foi o que apresentou as maiores médias de viscosidades.

Em um estudo de Adeleye e Rachal (2007) entre várias bebidas espessadas, como água, suco, café e leites, a com base láctea foi a mais viscosa, concordando com esse trabalho, segundo o qual as preparações com leite apresentaram as mais altas viscosidades quando comparadas com as da água e do suco.

No suco, a FA2 teve diferença significativa de todas as amostras $(p<0,05)$. Para o suco, quando classificado por consistências, os resultados foram semelhantes ao leite, porém a única diferença foi o amido de milho (AM), que atingiu a consistência "mel" (351-1750 cP).

Quanto à classificação de consistências em "ralo", "néctar", "mel" e "pudim" (Tabela 1) pode-se observar que o Espessante Comercial E.C2F, diluído conforme as orientações do fabricante, adquiriu a consistência "rala" (1-50 cP), não sendo adequado para pacientes com disfagia.

De acordo com Souza et al. (2003), os líquidos "ralos" dificultam a deglutição de pessoas que apresentam o controle oral reduzido e ainda aumentam o risco de aspiração do alimento. Germain et al. (2006) ao avaliarem a viscosidade de bebidas espessadas, tradicionalmente elaboradas com espessantes comerciais para disfagia, observaram que grande parte das amostras testadas não estavam de acordo com os níveis de consistência sugeridos pela National Dysphagia Diet (2002).

Mills (1996) relata que não há nenhuma garantia de que a viscosidade e consistência "néctar" ou "mel" de um fabricante seja semelhante quando comparadas a outros fabricantes, o que corrobora o presente trabalho, visto que os espessantes comerciais distintos E.C1F e E.C2F atingiram viscosidades e consistências diferentes.

De acordo com Garcia et al.(2005) vários fatores têm impacto sobre a viscosidade da amostra espessada, incluindo o tipo de agente espessante (por exemplo, amido ou goma), o líquido de base (como suco ou leite) e a sua temperatura. O leite, por exemplo, contém minerais e outros ingredientes que podem interagir com o espessante resultando em maior capacidade de espessamento.

Para Sopade et al. (2008) as diferenças de viscosidades entre água, leite e suco para as mesmas amostras, parece ter ocorrido devido aos diferentes constituintes de cada amostra, que tem forte impacto nos resultados finais de viscosidade. Interações podem ocorrer entre o agente espessante e alguns constituintes das amostras como a presença de pectina (suco de laranja), íons (alta concentração no suco de laranja e leite) e ácidos (suco de laranja). Para Toneli et al. (2005), a pectina presente no suco de laranja atua também como um agente espessante, o que justifica a viscosidade de

Tabela 3. Média das viscosidades (cP) e classificação quanto à consistência de diferentes espessantes em água, leite e suco.

\begin{tabular}{|c|c|c|c|}
\hline \multirow{2}{*}{ Amostras } & \multicolumn{3}{|c|}{ Viscosidades (cP) } \\
\hline & Água & Leite & Suco \\
\hline E.C1F & $382^{\circ}(\mathrm{Mel})$ & 3276 bc (Pudim) & $2605^{\circ}$ (Pudim) \\
\hline E.C1 & $613^{\circ}(\mathrm{Mel})$ & 7190 a (Pudim) & $4138^{\text {b }}$ (Pudim) \\
\hline E.C2F & $49^{\circ}$ (Rala) & $192^{\text {d }}$ (Néctar) & $266^{\text {d }}$ (Néctar) \\
\hline E.C2 & $2551^{\text {b Pudim) }}$ & 5352 abc (Pudim) & $2416^{\circ}$ (Pudim) \\
\hline $\mathrm{FB}$ & 4478 a (Pudim) & $2990^{\circ}$ (Pudim) & $2157^{\circ}$ (Pudim) \\
\hline PD & $4492^{\text {a }}$ (Pudim) & 5567 ab (Pudim) & $2917^{\text {bc }}$ (Pudim) \\
\hline AM & $671^{\circ}(\mathrm{Mel})$ & $3041^{\circ}$ (Pudim) & $546^{d}(\mathrm{Mel})$ \\
\hline FA1 & $64^{\circ}$ (Néctar) & 289 d (Néctar) & $291^{\text {d }}$ (Néctar) \\
\hline FA2 & $3251^{\text {ab }}$ (Pudim) & 5384 abc(Pudim) & 7019 a (Pudim) \\
\hline
\end{tabular}

Médias seguidas pela mesma letra na vertical não apresentam diferença estatística significativa $(P>0,05)$. E.C1F: Espessante comercial 1 diluído conforme o fabricante. E.C1: Espessante comercial $1 \mathrm{com} 10 \mathrm{~g}$ para água e leite e $8 \mathrm{~g}$ para suco. E.C2F: Espessante comercial 2 diluído conforme o fabricante. E. C2: Espessante comercial $2 \mathrm{com} 10 \mathrm{~g}$ para água e leite e $8 \mathrm{~g}$ para suco. FB: Fécula de batata com $10 \mathrm{~g}$ para água e leite e $8 \mathrm{~g}$ para suco. PD: Polvilho doce com $10 \mathrm{~g}$ para água e leite e $8 \mathrm{~g}$ para suco. AM: Amido de milho com $10 \mathrm{~g}$ para água e leite e $8 \mathrm{~g}$ para suco. FA1: Farinha de arroz com $10 \mathrm{~g}$ para água e leite e $8 \mathrm{~g}$ para suco. FA2: Farinha de arroz com $20 \mathrm{~g}$ para água e leite e $16 \mathrm{~g}$ para suco. 
todas as amostras de espessantes com suco de laranja terem ficado mais viscosas do que em água.

Conforme Takizawa et al. (2004) a viscosidadede aparente do amido de mandioca é mais elevada do que a do amido de milho na mesma concentração, corroborando o presente estudo, visto que a viscosidade do polvilho doce, obteve valores mais altos quando comparado com a do amido de milho em água, leite e suco.

Para a disfagia classificada como leve deve-se utilizar estratégias durante a alimentação como manejo de posturas e mais atenção à deglutição. Entretanto, já pode haver aspiração de líquidos "ralos", fato que contribui para que a consistência adequada para líquidos seja "néctar". De acordo com a Tabela 3 para água, leite e suco, a amostra FA1 atingiu esse tipo de consistência, já a E.C2F se mostra adequada apenas quando diluída em leite e suco. Segundo Costa et al. (2003), na disfagia moderada há aspiração do alimento com ausência de reflexos, por isso os líquidos devem ser engrossados a partir da consistência "mel". Nesse tipo de consistência, para água os tratamentos E.C1F, E.C1 e A.M estariam adequados; para leite, nenhuma das amostras foi classificada como "mel"; e, para sucos, a amostra AM está apropriada (546 cP). Logemann et al.(2008), afirmam que o espessamento de fluidos orais para consistência como "néctar" ou "mel" retarda o tempo de trânsito e pode ser mais fácil de controlar a deglutição, diminuindo o risco de aspiração para alguns.

Para Costa et al. (2003), na disfagia moderada grave, usa-se parcialmente a via oral, e a dieta deve ser de consistência "pudim". Nesses casos, para água, leite e suco, os tratamentos adequados foram o E.C2, a FB, o PD e a FA2. Além desses, apenas para leite e suco os tratamentos E.C1F e E.C1 também se mostraram adequados para pacientes com disfagia moderada grave. Segundo Costa et al. (2003), na disfagia grave, a alimentação via oral deve ser suspensa. Foram selecionadas para a avaliação sensorial as cinco amostras que se apresentaram mais promissoras e com viscosidade segura para pacientes com disfagia leve, moderada e moderada grave.

A Tabela 4 apresenta os resultados da análise sensorial da água, leite e suco com os diferentes tipos de formulações de espessantes.

As diferentes amostras de espessantes em água demonstraram que para o atributo aparência, o espessante comercial E.C1F, diluído conforme as orientações do fabricante, obteve $(6,56)$ de nota conforme a escala hedônica. Ainda na Tabela 4, em relação à escala hedônica de 9,0 pontos (ancorada do "desgostei muitíssimo" ao "gostei muitíssimo"), a aparência e o odor da amostra E.C1F atingiram a média de "gostei ligeiramente" (6,0 pontos na escala hedônica). Nas demais amostras para os mesmos atributos foram classificados como "nem gostei/nem desgostei" (PD) e "desgostei ligeiramente" (FB, AM, FA2). Para a consistência, todas as amostras não atingiram médias de "gostei".

Para sabor, a amostra E.C1F, que é um espessante comercial, recebeu a maior média $(5,33)$ que equivale ao "nem gostei/nem desgostei" na escala hedônica e

Tabela 4. Médias das notas da análise sensorial das diferentes amostras de espessantes em água, leite e suco.

\begin{tabular}{|c|c|c|c|c|c|}
\hline \multicolumn{6}{|c|}{ Tratamentos } \\
\hline Atributos & E.C1F & FB & PD & AM & FA \\
\hline \multicolumn{6}{|c|}{ Água } \\
\hline Aparência & $6,56^{a}$ & $4,86^{b}$ & $5,03 a b$ & $4,13^{b}$ & $4,10^{b}$ \\
\hline Consistência & $5,63^{a}$ & $3,83 \mathrm{~b}$ & $3,13^{b}$ & 4,66 ab & $4,30 \mathrm{ab}$ \\
\hline Odor & $6,03^{a}$ & $5,70 a b$ & $5,96^{a}$ & $4,53^{b}$ & $5,26 \mathrm{ab}$ \\
\hline Sabor & $5,33^{a}$ & $3,90 a b$ & $4,96^{a}$ & $2,96^{b}$ & $4,26 \mathrm{ab}$ \\
\hline Aceitação Global & $5,80^{a}$ & $4,40 a b$ & $4,23^{b}$ & $3,40^{b}$ & $4,50 \mathrm{ab}$ \\
\hline \multicolumn{6}{|c|}{ Leite } \\
\hline Aparência & $5,50 a b$ & $5,80^{a}$ & $4,46^{b}$ & 5,66 ab & $5,30 \mathrm{ab}$ \\
\hline Consistência & $5,56^{a}$ & $6,03^{a}$ & $2,86^{b}$ & $5,06^{a}$ & $4,90^{a}$ \\
\hline Odor & $5,40^{a}$ & $5,26^{a}$ & $5,03^{a}$ & $5,03^{a}$ & $5,46^{a}$ \\
\hline Sabor & $5,20^{a}$ & $4,30^{a}$ & $4,60^{a}$ & $4,06^{a}$ & $4,40^{a}$ \\
\hline Aceitação Global & $5,26^{a}$ & $4,63 a b$ & $3,73^{b}$ & $4,46 \mathrm{ab}$ & $4,46^{a b}$ \\
\hline \multicolumn{6}{|c|}{ Suco } \\
\hline Aparência & $5,73^{a}$ & $5,16 a b$ & $6,16^{a}$ & $5,70^{\text {a }}$ & $4,10^{b}$ \\
\hline Consistência & $5,93^{a}$ & $3,93^{\text {a }}$ & $4,06^{a}$ & $4,60^{\text {a }}$ & $3,56^{\text {a }}$ \\
\hline Odor & $6,63^{a}$ & $5,70 a b$ & $5,83 a b$ & $5,20^{b}$ & $5,06^{b}$ \\
\hline Sabor & $6,13^{a}$ & $5,13 a b$ & $5,20 \mathrm{ab}$ & $4,50^{b}$ & $3,70^{b}$ \\
\hline Aceitação Global & $5,76^{a}$ & $5,13 \mathrm{ab}$ & $5,10 \mathrm{ab}$ & $4,63^{a b}$ & $3,80^{b}$ \\
\hline
\end{tabular}

Médias seguidas pela mesma letra na horizontal não apresentam diferença estatística significativa $(P>0,05)$. E.C1F: Espessante comercial 1. AM: Amido de milho. FA: Farinha de arroz. FB: Fécula de batata. PD: Polvilho doce. 
a que recebeu a menor nota foi a amostra elaborada com o espessante $\operatorname{AM}(2,96)$ que sugere classificação "desgostei muito" pela escala hedônica.

Conforme a Tabela 4, para o atributo aparência, a melhor nota foi a do polvilho doce com classificação "gostei ligeiramente" $(6,16)$, opondo-se ao resultado com o leite. Quanto à consistência a melhor classificação ficou em "nem gostei/nem desgostei" com a amostra E.C1F, assim como para o atributo odor e sabor. Quanto à classificação global, E.C1F $(5,76), \operatorname{FB}(5,13)$ e PD $(5,10)$ também ficaram com média 5,0 pela escala hedônica.

Como a análise sensorial foi realizada com universitários oriundos do curso de Nutrição e de Medicina e não portadores de disfagia, os avaliadores podem ter tido um grau elevado de exigência em relação às amostras oferecidas, o que pode justificar as baixas notas recebidas em todos os atributos. No estudo de Macqueen et al. (2003) com indivíduos com e sem disfagias, foi constatado que não houve diferença estatística significativa entre esses dois grupos quando comparado o atributo sabor dos espessantes comerciais analisados. Na prática de nutrição clínica, Horwarth et al. (2005) orientam que existe a necessidade de se aumentar a qualidade de líquidos espessos, uma vez que as modificações da dieta continuam sendo uma intervenção comum para pacientes com disfagia.

Para Moura e Aschieri (2009), em géis é comum o aparecimento de uma película superficial, devido à retrogradação, o que compromete a qualidade do produto, principalmente no aspecto aparência, e pode explicar no presente trabalho o fato de o atributo aparência não ter atingido médias a partir de sete pontos da escala hedônica, equivalente a "gostei moderadamente". Lotong et al. (2003) afirmam que além de produzir uma consistência mais grossa, espessantes comerciais alteram características sensoriais das bebidas de base, como o sabor e a textura, o que pode tornar o líquido engrossado menos desejável para ingestão. Em um estudo de Wendin et al. (2010) os fluidos de alta viscosidade, que continham um agente espessante à base de amido, foram percebidos como mais fáceis de engolir e mais cremosos do que fluidos não espessados. Sousa et al. (2011) observaram que as dietas com mudanças na consistência são as que possuem maiores índices de resto-ingestão da dieta hospitalar, o que reforça a relevância do presente estudo quanto a consistência das amostras apenas terem atingido seis pontos na escala hedônica, resultando em "gostei ligeiramente".

Segundo Macqueen et al. (2003) muitos indivíduos com disfagia não gostam de fluidos engrossados, levando a uma baixa adesão de espessantes. Isso pode aumentar o risco de redução da ingestão diária de líquidos, de desidratação e de aspiração. Esse mesmo autor menciona ainda que quatro pacientes relataram que não
Tabela 5. Espessantes comerciais e domésticos utilizados nas análises com seus respectivos valores energéticos (kcal) e custos $(\$)$.

\begin{tabular}{cccc} 
Amostras & $\begin{array}{c}\text { Preço por } \\
\mathbf{1 0 0 g} \mathbf{( R \$ )}\end{array}$ & $\begin{array}{c}\text { Quantidade } \\
(\mathbf{g})\end{array}$ & $\begin{array}{c}\text { Valor energético } \\
\text { (kcal) }\end{array}$ \\
\hline EC1.F & 47,00 & 10 & 35 \\
EC2.F & 13,00 & 10 & 35 \\
AM & 1,64 & 10 & 34 \\
FA & 0,55 & 10 & 36,5 \\
FB & 2,32 & 10 & 32 \\
PD & 0,77 & 10 & 35 \\
\hline
\end{tabular}

E.C1F: Espessante comercial 1. E.C2F: Espessante comercial 2. AM: Amido de milho. FA: Farinha de arroz. FB Fécula de batata. PD: Polvilho doce.

conseguiam mais beber líquidos como suco de maçã, suco de laranja, chá, café e água. Isso revela a atenção que se deve ter na disfagia, visto que outros problemas de saúde podem ser bastante agravados pela falta da ingestão de líquidos.

Conforme a Tabela 5 os espessantes comerciais são mais onerosos, além de serem mais difíceis de adquirir. $\mathrm{O}$ uso do agente espessante doméstico pode ser mais econômico e conveniente. No entanto, requer precaução na utilização e habilidade do manipulador, visto que não existe uma normatização para a viscosidade ou consistência de receitas que utilizam espessantes domésticos (HORWARTH et al., 2005).

\section{Conclusões}

A viscosidade de um dos espessantes comerciais (E.C2F) não estava adequada para o consumo de pessoas disfágicas, obtendo consistência "rala".

O espessante comercial E.C1F apresentou consistência "mel", assim como o amido de milho (AM), sendo apropriado para o consumo de líquidos.

As amostras com leites foram as que apresentaram maiores viscosidades, a maioria obteve consistência "pudim".

As amostras de espessantes em suco foram semelhantes às do leite para o atributo consistência.

Em relação à aceitabilidade para água e suco, o espessante comercial E.C1F atendeu às expectativas em todos os atributos sensoriais. Em leite, as amostras E.C1F juntamente com a fécula de batata (FB) foram as que mais se destacaram sensorialmente.

\section{Referências}

ADELEYE, B.; RACHAL, C. Comparison of the rheological properties of ready-to-service and powdered instant food-thickened beverages at different temperatures for dysphagic patients. Journal of the Academy of Nutrition and Dietetics, Chicago, v. 107, n. 7, p. 11761182, 2007. 
Avaliação reológica e sensorial de espessantes domésticos em diferentes líquidos como alternativa na disfagia

SCHMIDT, H. e OLIVEIRA, V. R.

BRASIL. Conselho Nacional de Saúde. Resolução n 466, de 12 de dezembro de 2012. Diretrizes e normas regulamentadoras de pesquisa em seres humanos. Diário Oficial da República Federativa do Brasil, Brasília, DF, 13 jun. 2013. Seção 1, p. 59. Disponível em: <http://conselho.saude.gov.br/resolucoes/2012/Reso466.pdf>. Acesso em: 16 set. 2013.

COSTA, H. M.; LUIZ, M. O. R.; CARMONA, M. J. C.; CARDOSO, E.; ISOSAKI, M.; AULER JUNIOR, J. O. C. Reintrodução da alimentação oral em pacientes traqueostomizados com terapia de nutrição enteral. Revista Brasileira de Nutrição Clínica, São Paulo, v. 18, n. 4, p. 168-172, 2003.

CRARY, M. A.; MANN, G. D.; GROHER, M. E. Initial psychometric assessment of a functional oral intake scale for dysphagia in stroke patients. Archives of Physical Medicine and Rehabilitation, Philadelphia, v. 86, n. 8, p. 1516-1520, 2005. http://dx.doi.org/10.1016/j. apmr.2004.11.049. PMid:16084801

ESTAT. Sistema de análise estatística. versão 2.0. Jaboticabal: UNESP, 1992

FONTAN. G. C. R. Influência do uso de espessantes nas características sensoriais e físico-químicas de bebida láctea com polpa de umbu. 2008. 57 f. Dissertação (Mestrado em Engenharia de Alimentos)-Universidade Estadual do Sudoeste da Bahia, Itapetinga, 2008.

GARCIA, J. M.; CHAMBERS, E. IV; MATTA, Z.; CLARK, M. Viscosity measurements of nectar- and honey-thick liquids: product, liquid, and time comparisons. Dysphagia, New York, v. 20, n. 4, p. 325-335, 2005. http://dx.doi.org/10.1007/s00455-005-0034-9. PMid:16633878

GERMAIN, I.; DUFRESNE, T.; GRAY-DONALD, K. A novel dysphagia diet improves the nutrient intake of institutionalized elders. Journal of the American Dietic Association, Chicago, v. 106, n. 10, p. 1614-1623, 2006

HORWARTH, M.; BALL, A.; SMITH, R. Taste preference and rating of commercial and natural thickeners. Rehabilitation nursing :the official journal of the Association of Rehabilitation Nurses, Hoboken, v. 30, n. 6, p. 239-246, 2005. http://dx.doi. org/10.1002/j.2048-7940.2005.tb00118.x. PMid:16294803

LOGEMANN, J. A.; GENSLER, G.; ROBBINS, J.; LINDBLAD, A. S.; BRANDT, D.; HIND, J. A.; KOSEK, S.; DIKEMAN, K.; KAZANDJIAN, M.; GRAMIGNA, G. D.; LUNDY, D.; MCGARVEY-TOLER, S.; MILLER GARDNER, P. J. A randomized study of three interventions for aspiration of thin liquids in patients with dementia or Parkinson's disease. Journal of Speech, Language, and Hearing Research: JSLHR, Rockville, v. 51, n. 1, p. 173-183, 2008. http://dx.doi. org/10.1044/1092-4388(2008/013). PMid:18230864

LOTONG, V.; CHUN, S. S.; CHAMBERS, E.; GARCIA, J. M. Texture and flavor characteristics of beverages containing commercial thickening agents for dysphagia diets. Journal of Food Science, Chicago, v. 68, n. 4, p. 1537-1541, 2003. http://dx.doi.org/10.1111/j.1365-2621.2003. tb09680.x

MACQUEEN, C.; TAUBERT, S.; COTTER, D.; STEVENS, S.; FROST, G. Which commercial thickening agent do patients prefer? Dysphagia,
New York, v. 18, n. 1, p. 46-52, 2003. http://dx.doi.org/10.1007/s00455002-0084-1. PMid:12497196

MILLS, R. H. Rheology overview: control of liquid viscosities in dysphagia management. Nutrition in Clinical Practice, Dallas, v. 14, p. 52-56, 1996. Suplemento.

MOURA, L. S. M.; ASCHERI, J. L. R. Efeitos das variáveis de extrusão sobre propriedades de pasta de farinhas mistas pré-gelatinizadas de arroz (Oryza sativae, L.), feijão (Phaseolus vulgaris L.) e milho (Zea mays L.). Alimentos e Nutrição, Araraquara, v. 24, n. 1, p. 101-114, 2013.

NATIONAL DYSPHAGIA DIET. Standardization for optimal care. Chicago: American Dietetic Association, 2002.

SCHELP, A. O.; COLA, P. C.; GATTO, A. R.; SILVA, R. G.; CARVALHO, L. R. Incidência de disfagia orofaríngea após acidente vascular encefálico em hospital público de referência. Arquivos de NeuroPsiquiatria, São Paulo, v. 62, n. 2B, p. 503-506, 2004. http://dx.doi. org/10.1590/S0004-282X2004000300023. PMid:15273852

SILVA, L. B. C.; IKEDA, C. M. Cuidado nutricional na disfagia: uma alternativa para maximização do estado nutricional. Revista Brasileira de Nutrição Clínica, São Paulo, v. 24, n. 3, p. 203-210, 2009

SOCIEDADE BRASILEIRA DE GERIATRIA E GERONTOLOGIA SBGG. I Consenso Brasileiro de nutrição e disfagia em idosos hospitalizados. Barueri: Manole, 2011.

SOPADE, P. A.; HALLEY, P. J.; CICHERO, J. A. Y.; WARD, L. C.; LIU, J.; VARLIVELI, S. Rheological characterization of food thickeners marketed in Australia in various media for the management of dysphagia. III: Fruit juice as a dispersing medium. Journal of Food Engineering, Essex, v. 86, n. 4, p. 604-615, 2008. http://dx.doi. org/10.1016/j.jfoodeng.2007.11.013.

SOUSA, A. A.; GLORIA, M. S.; CARDOSO, T. S. Aceitação de dietas em ambiente hospitalar. Revista de Nutrição, Campinas, v. 24, n. 2, p. 287-294, 2011. http://dx.doi.org/10.1590/S1415-52732011000200009.

SOUZA, B. B. A.; MARTINS, C.; CAMPOS, D. J.; BALSINI, I. D.; MEYER, L. R. A. Nutrição e disfagia: guia para profissionais. Curitiba: Nutro Clínica, 2003

TAKIZAWA, F. F.; SILVA, G. O.; KONKEL, F. E.; DEMIATE, I. M. Characterization of tropical starches modified with potassium permanganate and lactic acid. Brazilian Archives of Biology and Technology, Curitiba, v. 47, n. 6, p. 921-931, 2004. http://dx.doi. org/10.1590/S1516-89132004000600012.

TONELI, J. T. C. L.; MURR, F. E. X.; PARK, K. J. Review: estudo da reologia de polissacarídeos utilizados na indústria de alimentos. Revista Brasileira de Produtos Agroindustriais, Campina Grande, v. 7, n. 2, p. 181-204, 2005.

UNIVERSIDADE ESTADUAL DE CAMPINAS - UNICAMP. Tabela Brasileira de Composição de Alimentos - TACO. Versão 2. 2.ed. Campinas: NEPA; UNICAMP, 2006. 113 p.

WENDIN, K.; EKMAN, S.; STADING, M. Objective and quantitative definitions of modified food textures based on sensory and rheological methodology. Food \& Nutrition Research, Bålsta, v. 54, p. 51345144, 2010 